\title{
Actions Speak Louder than Words: Proving Bisimilarity for Context-Free Processes
}

\author{
Hans Hüttel - hans@cs . auc.dk* \\ Department of Computer Science \\ Aalborg University \\ Fredrik Bajersvej 7E \\ 9220 Aalborg $\varnothing$ \\ DENMARK \\ Colin Stirling - cps@dcs.ed.ac.uk \\ Laboratory for the Foundations of Computer Science \\ James Clerk Maxwell Building \\ University of Edinburgh \\ Edinburgh EH9 3JZ \\ SCOTLAND
}

October 1997

\begin{abstract}
Baeten, Bergstra, and Klop (and later Caucal) have proved the remarkable result that bisimulation equivalence is decidable for irredundant context-free grammars. In this paper we provide a much simpler and much more direct proof of this result using a tableau decision method involving goal-directed rules. The decision procedure also provides the essential part of the bisimulation relation between two processes which underlies their equivalence. We also show how to obtain a sound and complete sequent-based equational theory for such processes from the tableau system and how one can extract what Caucal calls a fundamental relation from a successful tableau.
\end{abstract}

\footnotetext{
${ }^{*}$ An earlier shorter version of this paper appeared at the 6th Annual Symposium on Logic in Computer Science (LICS 91), Amsterdam 1991.
} 


\section{Introduction}

In [1] (and [2]) Baeten, Bergstra, and Klop prove the remarkable result that bisimulation equivalence is decidable for irredundant context-free grammars (without the empty production). Within process calculus theory these grammars correspond to normed processes defined by a finite family of guarded recursion equations in the signature of BPA (Basic Process Algebra) [4]. These processes can have infinitely many states (even after quotienting by bisimulation equivalence). Consequently the process calculus approach (as exemplified in [27]) encompasses a much richer class of infinite-state systems that are open to automatic techniques normally associated with finite state systems than all those approaches based on trace, or language, equivalence. Recently, Huynh and Tian [22] have shown that failures and readiness equivalences are undecidable for this class of processes and Groote and the first author [17] have proved that in fact all known equivalences other than bisimulation are undecidable here, thus suggesting a new criterion for distinguishing between the computational qualities of behavioural equivalences.

However, the proof of decidability in $[1,2]$ is not easy as it relies on isolating a possibly complex periodicity from the transition graphs of these processes. An alternative, more elegant, proof utilizing rewrite techniques is presented by Caucal [7]; a simplified version of this proof is due to Groote [16]. The idea is to show that the maximal bisimulation on a transition graph is given as the least congruence of a canonical and strongly normalizing Thue system and that there are only finitely many candidates for such a system. However, the decision procedure consists of a linear search for the desired Thue system. Neither of the proofs reflects how one intuitively would show that two processes are bisimilar.

In this paper we first present a simpler and much more direct proof of the decidability result using a tableau decision method. The tableau method attempts to construct a tableau, a proof tree constructed in a goal-directed fashion. One starts with a goal equation $E=F$ which is then broken down into subgoal equations by applying tableau proof rules. The tableau construction ends along some tree branch whenever certain termination criteria apply. Termination can be either successful or unsuccessful. In case of successful termination along all branches, the tableau is said to be successful. An important result is that the root equation $E=F$ is true if and only if one can construct a successful tableau starting from it. Another important result is that the tableau construction always terminates and that there are only finitely many possible tableaux for any given root equation. It is the conjunction of these two results that ensures decidability: a decision procedure

now consists in constructing all the finitely many tableaux and determining 
whether one of them is successful.

Our tableau method is closely related to the branching algorithms introduced by Korenjak and Hopcroft for the study of equivalence problems in language theory [24]. Indeed, the decision procedure for the equivalence of simple grammars [24] may be seen as a special case of our method. The tableau method is also related to the tableau methods used by the second author in the different context of local model checking finite and infinite state transition systems $[30,5]$. The decision procedure yields an upper bound on the depth of a tableau. Moreover, it provides the essential part of the bisimulation relation between two processes which underlies their equivalence, a self-bisimulation in the sense of [7].

An important by-product of the tableau system is a sound and complete sequent-based equational theory for normed BPA processes; the theory emanates from 'running the tableau method backwards'. This result extends Milner's axiomatization of regular processes [26] to the class of 'context-free' processes. At the same time it offers an alternative method for designing equational theories which does not depend on appealing to normal forms.

Since the first version of the present paper appeared, there have been a number of important results on the decidability of behavioural equivalences for processes with infinite transition graphs. We return to these results in the last section of our paper.

The rest of our paper is organized as follows: Preliminaries are dealt with in Section 2. In Section 3 we present the tableau decision method and give an upper bound on the depth of a tableau. Section 4 gives a sound and complete equational theory for these normed context-free processes that follows from the tableau system and finally, in Section 5 we show how to extract what Caucal calls a fundamental relation from a successful tableau.

\section{Preliminaries}

\subsection{Normed recursive BPA processes}

We consider the class of guarded recursive normed BPA (Basic Process Algebra) processes (see e.g. $[2,4]$ ). BPA process expressions are given by the abstract syntax

$$
E::=a|X| E_{1}+E_{2} \mid E_{1} E_{2}
$$

Here $a$ ranges over a set of atomic actions, and $X$ over a set of variables. The operator + is nondeterministic choice while $E_{1} E_{2}$ is the sequential com- 
position of $E_{1}$ and $E_{2}$. A process is defined by a finite system of recursive process equations $\Delta$

$$
\Delta=\left\{X_{i} \stackrel{\text { def }}{=} E_{i} \mid 1 \leq i \leq k\right\}
$$

where the $X_{i}$ are distinct, and the $E_{i}$ are guarded BPA expressions with free variables in $\operatorname{Var}=\left\{X_{1}, \ldots, X_{m}\right\}$. Guarded expressions are defined as follows: $a$ is always guarded. The sum $E_{1}+E_{2}$ is guarded, whenever $E_{1}$ and $E_{2}$ are. The sequential composition $E_{1} E_{2}$ is guarded, whenever $E_{1}$ is. Intuitively, a process expression is guarded if every variable occurrence is only accessible after an atomic action has been performed.

In the sequel we let $X, Y, \ldots$ range over variables in $\operatorname{Var}$ and $\alpha, \beta, \ldots$ over finite length sequences of variables.

Given a system of process equations $\Delta$ we define its transition behaviour by the rules given below. Note that $\epsilon$ is not a BPA expression according to our grammar; $\epsilon$ occurs as a configuration in the operational semantics to describe the end result of performing an atomic action. Further, we shall define $\epsilon$ to be the neutral element w.r.t. sequential composition. In other words, $\epsilon F$ is just $F$.

$$
\begin{array}{cr}
\frac{E \stackrel{a}{\rightarrow} E^{\prime}}{E+F \stackrel{a}{\rightarrow} E^{\prime}} & \frac{F \stackrel{a}{\rightarrow} F^{\prime}}{E+F \stackrel{a}{\rightarrow} F^{\prime}} \\
\frac{E \stackrel{a}{\rightarrow} E^{\prime}}{E F \stackrel{a}{\rightarrow} E^{\prime} F} & a \stackrel{a}{\rightarrow} \epsilon \quad a \in A c t \\
\frac{E \stackrel{a}{\rightarrow} E^{\prime}}{X \stackrel{a}{\rightarrow} E^{\prime}} & X \stackrel{\text { def }}{=} E \in \Delta
\end{array}
$$

A simple extension is the transitive closure of the relations $\{\stackrel{a}{\rightarrow} \mid a \in$ Act $\}$ : for $w \in A c t^{+}$we write $p \stackrel{a w}{\rightarrow} q$ if $p \stackrel{a}{\rightarrow} p^{\prime}$ and $p^{\prime} \stackrel{w}{\rightarrow} q$ for some $p^{\prime}$.

The important extra restriction on a system of process equations $\Delta$ is normedness.

Definition 1 [2] The norm of a BPA expression $E$ is

$$
|E|=\min \{\operatorname{length}(w) \mid E \stackrel{w}{\rightarrow} \epsilon\}
$$

$E$ is said to be normed if $|E|<\infty$. If for all variables $X$ in $\Delta$ we have $|X|<\infty$ we say that $\Delta$ is normed. 


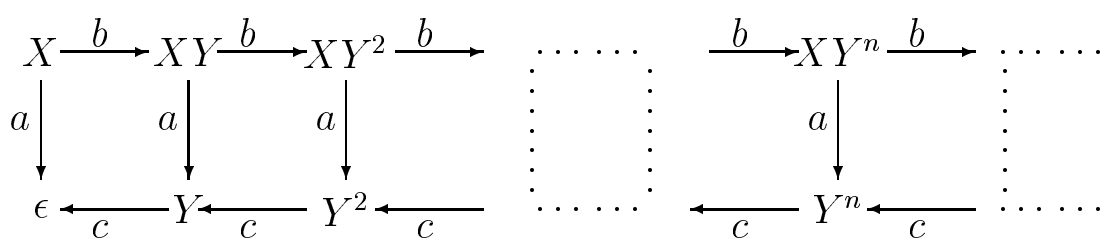

Figure 1: Transition graph for $X \stackrel{\text { def }}{=} a+b X Y ; \quad Y \stackrel{\text { def }}{=} c$ (Example 1)

The norm is easily computed: we have $|a|=1,|E+F|=\min (|E|,|F|)$, $|E F|=|E|+|F|$ and when $X \stackrel{\text { def }}{=} E,|X|=|E|$.

Example 1 Consider the pair $X \stackrel{\text { def }}{=} a+b X Y ; \quad Y \stackrel{\text { def }}{=} c$. Here $|X|=|Y|=1$. By the transition rules above $X$ generates the transition system in Figure 1 .

Because of the normedness restriction, this class of processes does not include the regular processes (such as $X \stackrel{\text { def }}{=} a X$ ). Nevertheless, it is a very rich class of processes and as illustrated in Figure 1, it contains processes that can have infinitely many states even after quotienting by bisimulation equivalence.

The language $L\left(X_{i}\right)$ accepted by a variable $X_{i}$ is the set $\left\{w \mid X_{i} \stackrel{w}{\rightarrow} \epsilon\right\}$. For instance, in Example 1 above $L(Y)=\{c\}$ whereas $L(X)=\left\{b^{n} a c^{n} \mid n \geq 0\right\}$. (According to basic formal language theory, $b^{n} a c^{n}$ is a string consisting of $n$ occurrences of $b$ followed by an $a$ and $n$ occurrences of $c$.)

When the variables of two families $\Delta$ and $\Delta^{\prime}$ are disjoint, the system of process equations $\Delta \cup \Delta^{\prime}$ also defines a set of normed processes in normal form. Consequently in general the question of whether $L(X)=L(Y)$ when $X, Y$ are variables in a system of BPA process equations $\Delta$ is undecidable since this is just a reformulation of the usual equivalence problem for contextfree grammars [19].

\subsection{Bisimulation equivalence}

Within process calculus theory a variety of equivalences have been proposed in order to capture when two processes may be said to exhibit the same behaviour. The most notable is bisimulation equivalence [28], as utilized in [27] for example. 
Definition 2 A relation $R$ between processes is a bisimulation if whenever $p R q$ then for each $a \in A c t$

1. $p \stackrel{a}{\rightarrow} p^{\prime} \Rightarrow \exists q^{\prime}: q \stackrel{a}{\rightarrow} q^{\prime} \wedge p^{\prime} R q^{\prime}$

2. $q \stackrel{a}{\rightarrow} q^{\prime} \Rightarrow \exists p^{\prime}: p \stackrel{a}{\rightarrow} p^{\prime} \wedge p^{\prime} R q^{\prime}$

Two processes $p$ and $q$ are said to be bisimulation equivalent or bisimilar, written $p$ simq, if there is a bisimulation $R$ such that $p R q$.

Not only is sim an equivalence relation, but it is also a congruence relation w.r.t. the process constructs of BPA [4].

Example 2 For an example of bisimilar BPA process expressions take the system of process equations $\{X \stackrel{\text { def }}{=} a Y X+b, Y \stackrel{\text { def }}{=} b X, A \stackrel{\text { def }}{=} a C+b, C \stackrel{\text { def }}{=}$ $b A A\}$. We have that $X \operatorname{sim} A$; the reader may want to verify that the relation $\left\{\left(X^{n}, A^{n}\right) \mid n \geq 0\right\} \cup\left\{\left(Y X^{n+1}, C A^{n}\right) \mid n \geq 0\right\}$ is a bisimulation (where $X^{n}$ here denotes $n$ successive $X \mathrm{~s}, X \in V)$.

The following proposition, originally due to Caucal [8], is essential, providing us with a way of removing suffixes of bisimilar BPA expressions.

Proposition 1 If $G$ is a normed BPA process expression and $E, F$ are arbitrary BPA process expressions and $E G$ simF $G$ then $E$ sim $F$.

Proof: We show that the relation

$$
R=\{(E, F) \mid E G \operatorname{sim} F G \text { for some } G\} \cup\{(\epsilon, \epsilon)\}
$$

is a bisimulation. Suppose not. Then wlog $(E, F) \in R$ and for some $E^{\prime}$ which may be $\epsilon, E \stackrel{a}{\rightarrow} E^{\prime}$ and whenever $F \stackrel{a}{\rightarrow} F^{\prime}$ we have that $\left(E^{\prime}, F^{\prime}\right) \notin R$. We know that there is a normed $G$ such that $E G \operatorname{sim} F G$. We consider two cases. First $E^{\prime}=\epsilon$. Then $E G \stackrel{a}{\rightarrow} G$. By assumption, if $F \stackrel{a}{\rightarrow} F^{\prime}$ then $F^{\prime} \neq \epsilon$. Hence $F G \stackrel{a}{\rightarrow} F^{\prime} G$ for some $F^{\prime} \neq \epsilon$ such that $G \operatorname{sim} F^{\prime} G$. But this is impossible, as $|G| \neq\left|F^{\prime} G\right|$ and therefore $G / \operatorname{sim} F^{\prime} G$. Otherwise $E^{\prime} \neq \epsilon$ and so $E G \stackrel{a}{\rightarrow} E^{\prime} G$. Consequently $F G \stackrel{a}{\rightarrow} F^{\prime} G$ for some $F^{\prime}$ such that $E^{\prime} G \operatorname{sim} F^{\prime} G$. If $F^{\prime} \neq \epsilon$ then after all $\left(E^{\prime}, F^{\prime}\right) \in R$. If $F^{\prime}=\epsilon$ then $E^{\prime} G \operatorname{sim} G$ which again is impossible.

Note that the proof relies on normedness; a simple counterexample for the unnormed case uses process equations $\{X \stackrel{\text { def }}{=} a X, Y \stackrel{\text { def }}{=} a\}$, as $Y Y X \operatorname{sim} Y X$ but clearly $Y Y / \operatorname{sim} Y$. 


\subsection{Normed recursive BPA processes in Greibach Nor- mal Form}

Any system of process equations $\Delta$ has a unique solution up to bisimulation equivalence [3]. Moreover, in [2] it is shown that any guarded system of equations can be effectively presented in a normal form (2.3), using a system of process equations $\Delta^{\prime}$ of the form

$$
\left\{X_{i} \stackrel{\text { def }}{=} \sum_{j=1}^{n_{i}} a_{i j} \alpha_{i j} \mid 1 \leq i \leq m\right\}
$$

where each variable sequence $\alpha_{i j}$ has length of at most two. This normal form preserves bisimulation equivalence. Moreover, when $\Delta$ only contains normed processes, so does $\Delta^{\prime}$.

This normal form is called $3-G N F$ (3-Greibach Normal Form) by analogy with context-free grammars (without the empty production). There is an obvious correspondence between variables and non-terminals and actions and terminals, and each equation $X_{i} \stackrel{\text { def }}{=} \sum_{j=1}^{n_{i}} a_{i j} \alpha_{i j}$ can be viewed as the family of productions $\left\{X_{i} \rightarrow a_{i j} \alpha_{i j} \mid 1 \leq j \leq n_{i}\right\}$. Normedness corresponds to irredundancy in the grammar. Conversely, any context-free language (without the empty string) is generated by such a grammar (where some variable is designated as the start symbol).

An important advantage of using GNF is that the states in the transition graph for a process given in this way are elements of $\operatorname{Var}^{*}$. Moreover, the restriction to variable sequences of length at most 2 guarantees limited growth of these sequences under single transitions. When applying a defining equation to the leftmost variable in a string $\alpha$ the length of the derivative increases by at most 1 :

Proposition 2 Suppose $\Delta$ is in 3-GNF. Then, for any $\alpha \in V a r^{*}$, whenever $\alpha \stackrel{a}{\rightarrow} \alpha^{\prime}$ we have length $\left(\alpha^{\prime}\right) \leq$ length $(\alpha)+1$.

Proof: Suppose $\alpha=X_{i} \alpha^{\prime \prime \prime}$. Then $\alpha \stackrel{a}{\rightarrow} \alpha^{\prime}$ must be due to $X_{i} \stackrel{a}{\rightarrow} \alpha^{\prime \prime}$. This in turn is due to the defining equation $X_{i} \stackrel{\text { def }}{=} \sum_{j=1}^{n_{i}} a_{i j} \alpha_{i j}$ having a summand $a \alpha^{\prime \prime}$ with length $\left(\alpha^{\prime \prime}\right) \leq 2$. Since $\alpha^{\prime}=\alpha^{\prime \prime} \alpha^{\prime \prime \prime}$, the result follows.

Any normed system of process equations has a variable whose norm is maximal. We shall denote this norm by $m_{\Delta}$. With this in mind, there is a simple relationship between lengths and norms for variable sequences, which will be useful later on.

Proposition 3 Let $\Delta$ be a normed system of guarded BPA equations. Let $m_{\Delta}$ be the maximal norm of any variable in $\Delta$. For $\alpha \in \operatorname{Var}^{*}$ length $(\alpha) \leq|\alpha|$ and $|\alpha| \leq m_{\Delta}$ length $(\alpha)$. 
ProOF: As any variable in $\alpha$ has norm at least 1, the norm of $\alpha$ must be at least the length of $\alpha$. Moreover, as any variable in $\alpha$ contributes at most $m_{\Delta}$ to the total norm, the total norm can be at most $m_{\Delta}$ times the length of $\alpha$.

\subsection{Self-bisimulations}

For finite-state processes a naive decision procedure for the bisimulation problem $p$ simq consists in enumerating all binary relations over the state space and determining if there is a relation among them which is a bisimulation containing $(p, q)$. But since in general bisimulations over normed BPA processes may be infinite - for instance, the least non-empty bisimulation over pairs of processes in the transition graph of Example 1 is the identity relation - a decision procedure for the bisimulation problem for normed BPA cannot rely on this.

The tableau procedure presented in this paper proceeds by comparing strings of BPA variables and simplifying them according to a simple strategy that may involve the substitution of subexpressions from elsewhere in the tableau. Thus, we cannot expect the pairs of expressions that we encounter to constitute a bisimulation. We can, however, obtain a 'bisimulation up to simplification, concatenation and substitution of subexpressions' from the tableau. From formal language theory we know that two strings are equal up to simplification, concatenation and substitution under some relation $R$ if they are contained in the least congruence containing $R$.

So we are dealing with a notion of 'bisimulation up to least congruence' and it turns out that this notion of 'bisimulation up to' suffices. As we only consider strings of BPA variables, we shall only need to consider 'bisimulation up to sequential congruence' and do not need to involve the nondeterministic choice operator at all.

Such relations, introduced by Didier Caucal in [8] (originally published as [7]), are commonly referred to as self-bisimulations. Whenever $\alpha \operatorname{sim} \beta$, our tableau system will construct a finite self-bisimulation, a relation $R \subseteq$ $\operatorname{Var}^{*} \times \operatorname{Var}^{*}$ whose closure under congruence w.r.t. sequential composition is a bisimulation containing $(\alpha, \beta)$.

Definition 3 For any binary relation $R$ on Var*, $\vec{R}$ is the least precongruence w.r.t. sequential composition that contains $R, \overleftrightarrow{R}$ the symmetric closure of $\vec{R}$ and $\overleftrightarrow{R}$ * the reflexive and transitive closure of $\overleftrightarrow{R}$ and thus the least congruence w.r.t. sequential composition containing $R$. 
A self-bisimulation is then simply a bisimulation up to congruence w.r.t. sequential composition.

Definition 4 Let $\Delta$ be a normed system of BPA equations in $3-G N F$. A relation $R \subseteq \operatorname{Var}^{*} \times \operatorname{Var}^{*}$ is called a self-bisimulation iff $\alpha R \beta$ implies that

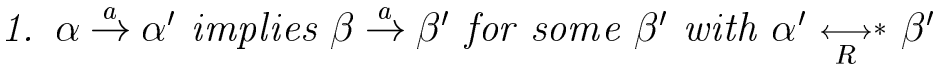

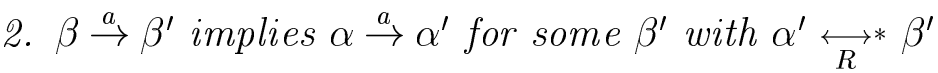

The following lemma, due to Caucal, shows that a self-bisimulation is a witness for bisimilarity.

Lemma 1 [8] If $R$ is a self-bisimulation then $\overleftrightarrow{R}^{*} \subseteq$ sim.

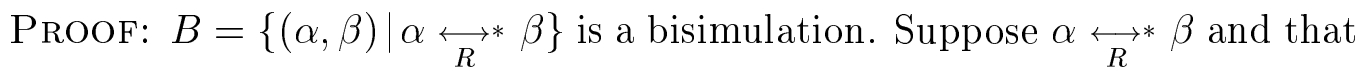

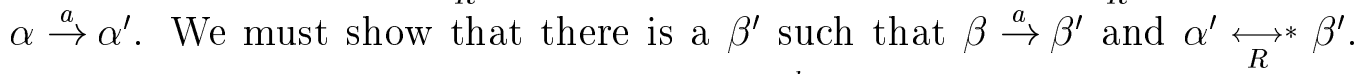

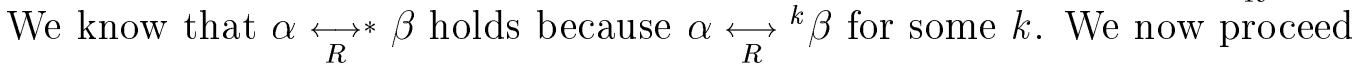
by induction in $k$.

$k=0$ : Trivial, for then $\alpha=\beta$.

$k=1$ : Either $\alpha \underset{R}{\rightarrow} \beta$ or $\beta \underset{R}{\rightarrow} \alpha$. Assume wlog that $\alpha \underset{R}{\rightarrow} \beta$. Then, by the definition of a least precongruence, there exists a $\left(\alpha_{0}, \beta_{0}\right) \in R$ such that $\alpha=\delta \alpha_{0} \gamma$ and $\beta=\delta \beta_{0} \gamma$. If $\delta \neq \epsilon, \alpha \stackrel{a}{\rightarrow} \alpha^{\prime}$ is due to $\delta \alpha_{0} \gamma \stackrel{a}{\rightarrow} \delta^{\prime} \alpha_{0} \gamma$, so our matching transition is $\delta \beta_{0} \gamma \stackrel{a}{\rightarrow} \delta^{\prime} \beta_{0} \gamma$; clearly $\delta^{\prime} \alpha_{0} \gamma \underset{R}{\longleftrightarrow} * \delta^{\prime} \beta_{0} \gamma$. If $\delta=\epsilon, \alpha \stackrel{a}{\rightarrow} \alpha^{\prime}$ is $\alpha_{0} \gamma \stackrel{a}{\rightarrow} \alpha_{1} \gamma$, due to $\alpha_{0} \stackrel{a}{\rightarrow} \alpha_{1}$. Since $\left(\alpha_{0}, \beta_{0}\right) \in R$, the

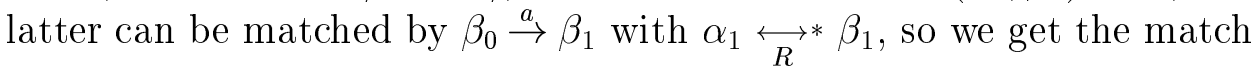
$\beta_{0} \gamma \stackrel{a}{\rightarrow} \beta_{1} \gamma$, and clearly $\alpha_{1} \gamma \underset{R}{\longleftrightarrow} * \beta_{1} \gamma$.

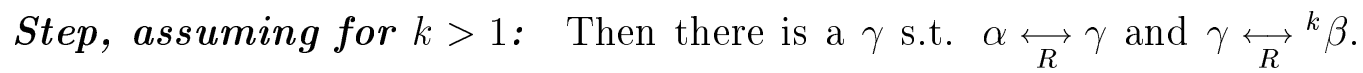
By induction hypothesis we know that there is a $\gamma^{\prime}$ s.t. $\gamma \stackrel{a}{\rightarrow} \gamma^{\prime}$ and $\alpha^{\prime} \overleftrightarrow{R} * \gamma^{\prime}$ and a $\beta^{\prime}$ s.t. $\beta \stackrel{a}{\rightarrow} \beta^{\prime}$ with $\gamma^{\prime} \overleftrightarrow{\alpha_{R}} * \beta^{\prime}$. But then by transitivity $\alpha^{\prime} \underset{R}{\overleftrightarrow{R}} * \beta^{\prime}$.

The other half of the proof, for $\beta \stackrel{a}{\rightarrow} \beta^{\prime}$, is identical.

Corollary $1 \alpha$ sim $\beta$ iff there is a self-bisimulation $R$ such that $\alpha R \beta$.

ProOf: Clearly, since sim is a congruence it is also a self-bisimulation. Conversely, by the above lemma, if $R$ is a self-bisimulation then $\overleftrightarrow{R} \underset{*}{*}$ is a bisimulation. 


\section{The tableau decision method}

The bisimulation checker for normed BPA we now present is a tableau system, a goal-directed proof system. The proof technique is similar to the algorithm used in [24] to show that language equivalence is decidable for simple grammars. See [15] for a further reference to this particular technique, known as branching algorithms in formal language theory.

The tableau method constructs a tableau, a proof tree constructed in a goal-directed fashion. The tableau construction starts with a goal equation $E=F$ which is then broken down into subgoal equations by applying tableau proof rules, and this same procedure is then applied to the subgoals in a recursive fashion. The tableau construction ends along some tree branch whenever certain termination criteria apply.

We assume a fixed system of normed BPA process equations in 3-GNF, $\Delta=\left\{X_{i} \stackrel{\text { def }}{=} \sum_{j=1}^{n_{i}} a_{i j} \alpha_{i j} \mid 1 \leq i \leq m\right\}$ where $m_{\Delta}$ is the maximal norm of any variable. We determine whether $X \alpha \operatorname{sim} Y \beta$ (assuming of course that all occurring variables are defined in $\Delta$ ) by constructing a tableau using the proof rules presented in Table 1.

A tableau for $X \alpha=Y \beta$ is a finite tree where all nodes are labelled with equations. $\alpha^{\prime}=\beta^{\prime}$. The root is labelled $X \alpha=Y \beta$ and the equations labelling the immediate successors of a node are determined by an application of one of the rules. A leaf of a tableau is a node which has no children. A tableau is maximal in the sense that every leaf is a terminal node as defined below. The tableau rules are not applied to terminal nodes.

The rules are built around equations $E \alpha=F \beta$ (where $\alpha, \beta$ could be the empty sequence of variables). Each rule has the form

$$
\frac{E \alpha=F \beta}{E_{1} \alpha_{1}=F_{1} \beta_{1} \cdots E_{n} \alpha_{n}=F_{n} \beta_{n}}
$$

(possibly with side conditions). The premise of a rule represents the goal to be achieved (that $E \alpha \operatorname{sim} F \beta$ ) while the consequents are the subgoals.

The rules are only applied to nodes that are not terminal. Terminal nodes are either successful or unsuccessful.

Definition 5 A tableau node is called an unsuccessful terminal if it has one of the forms

1. $\alpha=\beta$ with $|\alpha| \neq|\beta|$

2. $a \alpha=b \beta$ with $a \neq b$ 


\section{Rules within subtableaux}

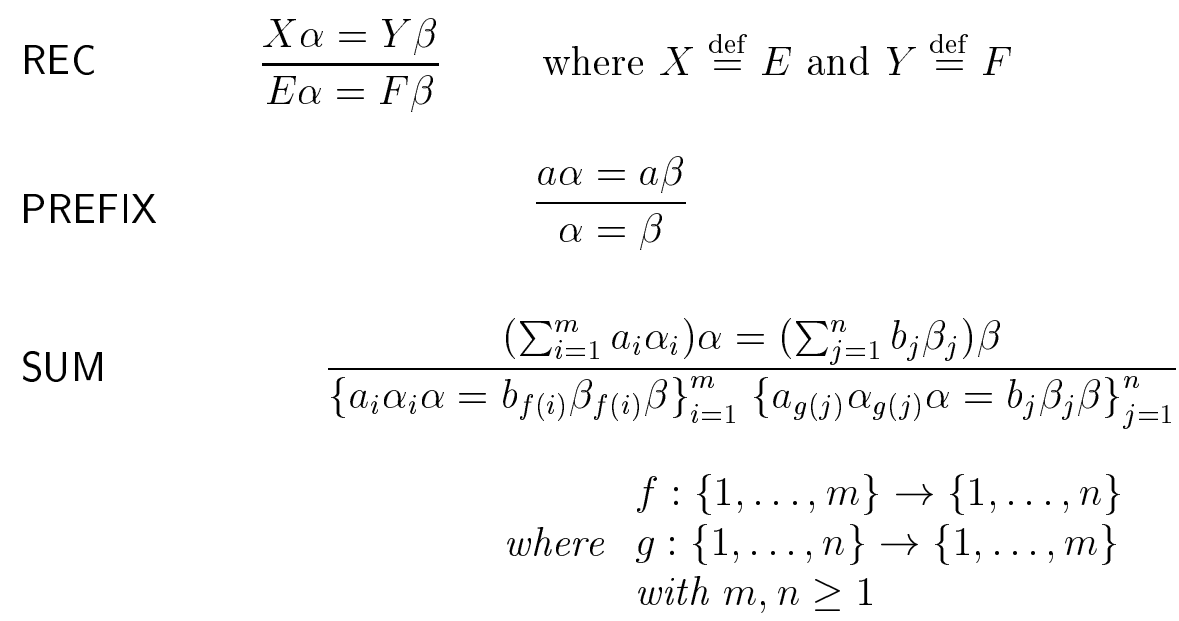

Rules for new subtableaux

SUBL $\quad \frac{\alpha_{i} \alpha=\beta_{i} \beta}{\alpha_{i} \gamma=\beta_{i}} \quad$ where $\alpha=\gamma \beta$ is the residual

SUBR $\quad \frac{\alpha_{i} \alpha=\beta_{i} \beta}{\alpha_{i}=\beta_{i} \gamma} \quad$ where $\gamma \alpha=\beta$ is the residual

Table 1: The tableau rules 
Clearly, such nodes cannot relate bisimilar processes. In the following subsection we define the notion of successful termination.

\subsection{Constructing subtableaux}

A tableau consists of a number of eliminating subtableaux constructed using the rules REC, SUM, and PREFIX of Table 1. Each of these rules is forwards sound in the following sense:

Proposition 4 (Soundness of REC, SUM and PREFIX)

- If $X \alpha \operatorname{sim} Y \beta$ and $X \stackrel{\text { def }}{=} E$ and $Y \stackrel{\text { def }}{=} F$, then $E \alpha \operatorname{sim} F \beta$.

- If $\left(\sum_{i=1}^{m} a_{i} \alpha_{i}\right) \alpha \operatorname{sim}\left(\sum_{j=1}^{n} b_{j} \beta_{j}\right) \beta$ then there exist functions $f:\{1, \ldots, m\} \rightarrow$ $\{1, \ldots, n\}$ and $g:\{1, \ldots, n\} \rightarrow\{1, \ldots, m\}$ such that $a_{i} \alpha_{i} \alpha \operatorname{simb}_{f(i)} \beta_{f(i)} \beta$ for $1 \leq i \leq m$ and $a_{g(j)} \alpha_{g(j)} \alpha \operatorname{simb}_{j} \beta_{j} \beta$ for $1 \leq j \leq n$.

- If a $\alpha \operatorname{sima} \beta$ then $\alpha \operatorname{sim} \beta$.

A subtableau is built from basic steps. See Figure 2.

Definition $6 A$ basic step for $X \alpha=Y \beta$ consists of an application of REC followed by at most one application of SUM followed by an application of PREFIX to each of its consequents (assuming that no node encountered is an unsuccessful terminal). A basic node is any node of the form $\alpha^{\prime}=\beta^{\prime}$ where $\alpha^{\prime}, \beta^{\prime} \in \operatorname{Var}^{*}$.

Corresponding to a basic step for $X \alpha=Y \beta$ is a set of single transition steps in the operational semantics, as $X \alpha \stackrel{a_{i}}{\rightarrow} \alpha_{i}$ and $Y \beta \stackrel{a_{i}}{\rightarrow} \beta_{i}$ for any consequent $\alpha_{i}=\beta_{i}$. By Proposition 2 we have that length $\left(\alpha_{i}\right) \leq 1+\operatorname{length}(X \alpha)$ and length $\left(\beta_{i}\right) \leq$ length $(Y \beta)+1$.

Suppose that $X \alpha=Y \beta$ and $|X| \leq|Y|$ and $|X|=k$. If we build a subtableau using $k$ basic steps, we will end up with an equation of the form $\alpha=\gamma \beta$. In other words, $X$ has been eliminated. A symmetric situation occurs, if $|Y| \leq|X|$. This is the idea behind the notion of an eliminating subtableau.

Definition 7 Assume that $k=\min (|X|,|Y|)$. An eliminating subtableau for $X \alpha=Y \beta$ is a subtableau which has been constructed using basic steps only and such that

- There are $k$ basic steps along all branches of the subtableau 
- If $|X| \leq|Y|$, then some leaf of the subtableau is labelled $\alpha=\gamma \beta$.

- If $|Y| \leq|X|$, then some leaf of the subtableau is labelled $\gamma \alpha=\beta$

Any leaf of the subtableau labelled $\alpha=\gamma \beta$ or $\gamma \alpha=\beta$ is called a residual.

See Figure 3 for a sketch of an eliminating subtableau in the case when $|X| \leq|Y|$. Notice that if $\alpha^{\prime}=\beta^{\prime}$ is a leaf of an eliminating subtableau then $X \alpha \stackrel{w}{\rightarrow} \alpha^{\prime}$ and $Y \beta \stackrel{w}{\rightarrow} \beta^{\prime}$ for some $w$ of length $k$.

In the case that $|X| \leq|Y|$ each leaf of an eliminating subtableau for $X \alpha=Y \beta$ is either a residual $\alpha=\gamma \beta$, as $X$ has been eliminated, or $\alpha_{i} \alpha=\beta_{i} \beta$ where $\alpha_{i}$ and $\beta_{i}$ need not be empty. Since the number of iterations of basic steps is $|X|$ there must be at least one residual and $\alpha$ and $\beta$ must persist as suffixes throughout the subtableau. For any such subtableau we pick one residual node and call it the residual. If instead $|Y|<|X|$ similar remarks apply.

The next step is to apply one of the SUB rules of Table 1 to each leaf other than residuals of an eliminating subtableau. If the residual is $\alpha=\gamma \beta$ we apply SUBL, and if it is $\gamma \alpha=\beta$ we apply SUBR. So assume $|X| \leq|Y|$; then for each leaf $\alpha_{i} \alpha=\beta_{i} \beta$ which is not a residual we obtain

$$
\frac{\alpha_{i} \alpha=\beta_{i} \beta}{\alpha_{i} \gamma=\beta_{i}} \quad \text { SUBL } \quad \text { where } \alpha=\gamma \beta \text { is the residual }
$$

If instead $|Y|<|X|$ so $\gamma \alpha=\beta$ is the residual, SUBR gives us the consequent $\alpha_{i}=\beta_{i} \gamma$. The SUB rules are also forwards sound in the following sense:

Proposition 5 (Soundness of SUBL and SUBR) If $\alpha_{i} \alpha \operatorname{sim} \beta_{i} \beta$ and $\alpha \operatorname{sim} \gamma \beta$ then $\alpha_{i} \gamma \operatorname{sim} \beta_{i}$. If $\gamma \alpha_{i} \operatorname{sim} \beta_{i}$ then $\alpha_{i} \operatorname{sim} \beta_{i} \gamma$.

ProOF: Since $\operatorname{sim}$ is a congruence, a substitution yields $\alpha_{i} \gamma \beta \operatorname{sim} \beta_{i} \beta$ and by Proposition 1 we get $\alpha_{i} \gamma=\beta$. The proof for the other half is entirely similar.

From the above proof we see that the SUB rules should be thought of as two-step rules consisting of a substitution using the residual followed by a reduction of the length of the expressions involved according to Proposition 1. For any application of SUB we have the following bounds on the sizes of the equations involved.

Proposition 6 For any eliminating subtableau with root $X \alpha=Y \beta$ and residual $\alpha=\gamma \beta$, for any leaf $\alpha_{i} \alpha=\beta_{i} \beta$ we have 
1. $|\alpha|<|X \alpha|$ and $|\gamma \beta|<|Y \beta|$

2. length $\left(\alpha_{i}\right)+$ length $(\gamma)+$ length $\left(\beta_{i}\right) \leq 3 m_{\Delta}+1$ for any application of SUB.

Proof: In an eliminating subtableau, we always choose to eliminate the variable with the least norm. Consequently the norm must decrease along any transition sequence leading to a residual. This gives us 1 . We assume that our systems of guarded BPA equations are in 3-GNF. So any basic step will at most increase the length of the variable sequences in the equations by 1 . As an eliminating subtableau has depth $\min (|X|,|Y|)$ the sequences $\alpha_{i}, \beta_{i}$ and $\gamma$ can each have length at most $\min (|X|,|Y|)$. Proposition 2 then gives us 2. Notice that the bound obtained here is completely independent of length $(\alpha)$ and length $(\beta)$.

We can now define successful termination.

Definition 8 A residual or a consequent of an application of a SUB rule is a successful terminal if it has one of the forms

1. $\alpha=\beta$ where there is a subtableau root above it also labelled $\alpha=\beta$.

2. $\alpha=\alpha$

It should be obvious that a node obeying termination condition 2 in the above relates bisimilar processes. It turns out that this is also true of termination condition 1 in the context of a successful tableau.

When a consequent of SUB or the residual is not a terminal node we build a new eliminating subtableau with it as root as described above, and continue in this fashion. Therefore, a tableau is defined as successions of eliminating subtableaux as sketched in Figure 4.

Definition 9 A successful tableau is a tableau all of whose leaves are successful terminals. If at any point in the construction of an eliminating subtableau we reach an unsuccessful terminal then the resulting tableau is unsuccessful.

Example 3 (Example 2 continued) Consider again $\Delta=\{X \stackrel{\text { def }}{=} a Y X+$ $b, Y \stackrel{\text { def }}{=} b X, A \stackrel{\text { def }}{=} a C+b, C \stackrel{\text { def }}{=} b A A\}$. The tableau in Figure 5 is a successful tableau for $X=A$. Note that the application of SUB to the equation $Y X=$ $C$ yields $Y X=C$, as the residual is here simply $\epsilon=\epsilon$. 


\subsection{Decidability, soundness, and completeness}

We now give the proof of correctness of the tableau method and give a complexity measure in terms of an upper bound on the length of a tableau path.

Theorem 1 Every tableau for $X \alpha=Y \beta$ is finite.

Proof: If a tableau were infinite then it would have an infinite path. By definition such a path could not contain either successful or unsuccessful terminals. By Proposition 6(2) such a path can not pass through infinitely many nodes which are consequents of a SUB rule, as there are only finitely many different equations whose components have norm $\leq 3 m_{\Delta}+1$ (by Proposition 3 ) the path would then contain some successful terminal infinitely often. Otherwise the path must almost always pass through a residual; but this also is impossible as the norm of a residual is strictly less than one directly above it by Proposition 6(1) and as the norms of the residuals are uniformly bounded by $\max (|\alpha|,|\beta|)$.

We can give a complexity bound on the tableau method in terms of the longest possible path in any tableau for $X \alpha=Y \beta$. We measure the length of a path in terms of the total number of basic steps, since this gives a measure of the number of transition matches that we need to consider.

Theorem 2 Any path in a tableau for $X \alpha=Y \beta$ has a length of at most

$$
m_{\Delta} \max \left(|\alpha|,|\beta|, m_{\Delta}\left\lceil\frac{3 m_{\Delta}+1}{2}\right\rceil \sum_{j=2}^{3 m_{\Delta}+1}(j-1) v^{j}\right)
$$

basic steps, where $v$ is the cardinality of Var.

Proof: Any SUB consequent has a length of at most $3 m_{\Delta}+1$, so there can be at most $\sum_{j=2}^{3 m_{\Delta}+1}(j-1) v^{j}$ distinct SUB consequents along a path. Between any two of these consequents there can be at most $\left\lceil\frac{3 m_{\Delta}+1}{2}\right\rceil$ residuals, since the worst that can happen is that the norm on each side decreases by 1 between two consecutive residuals. Thus, any containing SUB consequents has at most $\left\lceil\frac{3 m_{\Delta}+1}{2}\right\rceil \sum_{j=2}^{3 m_{\Delta}+1}(j-1) v^{j}$ subtableau roots. The leftmost path in a tableau contains only residuals, and since their norms are strictly decreasing there can be at most $\max (|\alpha|,|\beta|)$ residuals along this path. Since any subtableau can have a depth of at most $m_{\Delta}$ basic steps, the result follows.

Corollary 2 There are only finitely many tableaux for any $X \alpha=Y \beta$. 
Proof: This follows from the above theorem and the fact that the branching at any basic step in any tableau is uniformly bounded by the maximal number of SUM consequents. This is bounded by $2 B_{\Delta}$ where $B_{\Delta}=\max \left\{m \mid \exists X_{i} \in\right.$ $\left.\operatorname{Var}: X_{i} \stackrel{\text { def }}{=} \sum_{j=1}^{m} a_{i j} \alpha_{i j}\right\}$.

The next theorem states soundness and completeness of the tableau method. The proof of soundness relies on the notion of self-bisimulation introduced in section 2.4 .

Theorem $3 X \alpha \operatorname{sim} Y \beta$ iff there exists a successful tableau for $X \alpha=Y \beta$.

Proof: Suppose $X \alpha \operatorname{sim} Y \beta$. Then we can build a tableau for $X \alpha=Y \beta$ which has the property that for each node $\alpha^{\prime}=\beta^{\prime}$ we have $\alpha^{\prime} \operatorname{sim} \beta^{\prime}$. By Proposition 4 we can at any point in the tableau construction choose true consequents. By Theorem 1 this tableau construction must terminate and without unsuccessful terminals.

Now assume $\mathbf{T}$ is a successful tableau for $X \alpha=Y \beta$. We now show that $R_{\mathbf{T}}=\left\{(\alpha, \beta) \mid \alpha=\beta\right.$ is a basic node in $\left.\mathbf{T}, \quad \alpha, \beta \in \operatorname{Var}^{*}\right\}$ is a selfbisimulation. By Lemma 1 this means that $X \alpha \operatorname{sim} Y \beta$.

So suppose $\left(\alpha^{\prime}, \beta^{\prime}\right) \in R_{\mathbf{T}}$. We must then show that $\alpha^{\prime} \stackrel{a}{\rightarrow} \alpha^{\prime \prime}$ implies $\exists \beta^{\prime \prime}: \beta^{\prime} \stackrel{a}{\rightarrow} \beta^{\prime \prime}$ with $\alpha^{\prime \prime} \underset{R_{\mathbf{T}}}{\longrightarrow} \beta^{\prime \prime} . \alpha^{\prime}=\beta^{\prime}$ can either be a terminal or an internal node.

Suppose $\alpha^{\prime}=\beta^{\prime}$ is a terminal. If it is a terminal because of condition 2 we can certainly match within $\underset{R_{\mathrm{T}}}{\longrightarrow}$, since the least congruence of any relation contains the identity. Otherwise, if $\alpha^{\prime}=\beta^{\prime}$ is a terminal is due to condition 1 , there is a previous occurrence of $\alpha^{\prime}=\beta^{\prime}$ as a subtableau root. Then we have the following basic step in $\mathbf{T}$ :

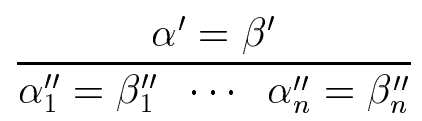

and $\alpha^{\prime} \stackrel{a_{i}}{\rightarrow} \alpha_{i}^{\prime \prime}$ is matched by $\beta^{\prime} \stackrel{a_{i}}{\rightarrow} \beta_{i}^{\prime \prime}$ because $\mathbf{T}$ is successful and $\left(\alpha_{i}^{\prime \prime}, \beta_{i}^{\prime \prime}\right) \in R_{\mathbf{T}}$ for $1 \leq i \leq n$ by definition of $R_{\mathbf{T}}$.

Otherwise $\alpha^{\prime}=\beta^{\prime}$ is an internal node. There are now two possibilities: either REC was applied to $\alpha^{\prime}=\beta^{\prime}$ or one of the SUB rules was.

Suppose REC was applied. Then we had the basic step

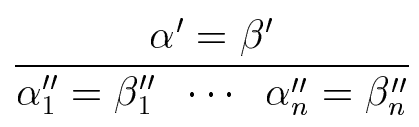


and just as in the above case, we can match within $R_{\mathbf{T}}$.

Now suppose a SUB rule was applied. Suppose wlog that it was SUBL. Further assume that $\alpha^{\prime}=\beta^{\prime}$ is $X_{1} \alpha_{1} \alpha_{0}=Y_{1} \beta_{1} \beta_{0}$, that $X_{1} \stackrel{\text { def }}{=} \sum_{i=1}^{m} a_{i} \alpha_{2 i}$ and $Y_{1} \stackrel{\text { def }}{=} \sum_{j=1}^{n} b_{j} \beta_{2 j}$ and that the residual was $\alpha_{0}=\gamma_{0} \beta_{0}$ :

$$
\frac{X_{1} \alpha_{1} \alpha_{0}=Y_{1} \beta_{1} \beta_{0}}{X_{1} \alpha_{1} \gamma_{0}=Y_{1} \beta_{1}} \text { SUBL }
$$

By definition $\left(\alpha_{0}, \gamma_{0} \beta_{0}\right) \in R_{\mathbf{T}}$. Either $X_{1} \alpha_{1} \gamma_{0}=Y_{1} \beta_{1}$ is a terminal or a subtableau root. In any case we must have that

$$
\forall a_{i}: X_{1} \alpha_{1} \gamma_{0} \stackrel{a_{i}}{\rightarrow} \alpha_{2 i} \alpha_{1} \gamma_{0} \exists b_{j}: b_{j}=a_{i}, Y_{1} \beta_{1} \stackrel{b_{j}}{\rightarrow} \beta_{2 j} \beta_{1} \text { with } \alpha_{2 i} \alpha_{1} \gamma_{0} \underset{R_{\mathbf{T}}}{\overleftrightarrow{\longrightarrow}} * \beta_{2 j} \beta_{1}
$$

If $X_{1} \alpha_{1} \gamma_{0}=Y_{1} \beta_{1}$ is a terminal, this follows from the same reasoning used in the case where $\alpha^{\prime}=\beta^{\prime}$ is a terminal. Otherwise, we have the basic step

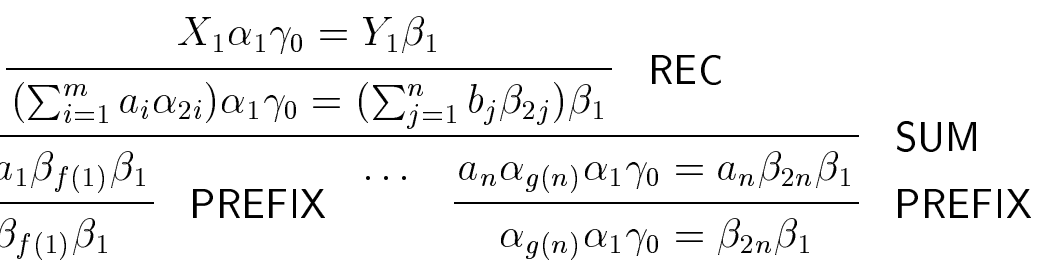

Now, what are the transitions of $X_{1} \alpha_{1} \alpha_{0}$ and $Y_{1} \beta_{1} \beta_{0}$ and how do we match them ? For $1 \leq i \leq m$ we have that $X_{1} \alpha_{1} \alpha_{0} \stackrel{a_{i}}{\rightarrow} \alpha_{2 i} \alpha_{1} \alpha_{0}$ and for $1 \leq j \leq n, Y_{1} \beta_{1} \beta_{0} \stackrel{b_{j}}{\rightarrow} \beta_{2 j} \beta_{1} \beta_{0}$. For any $X_{1} \alpha_{1} \alpha_{0} \stackrel{a_{i}}{\rightarrow} \alpha_{2 i} \alpha_{1} \alpha_{0}$ there is (1) a $Y_{1} \beta_{1} \beta_{0} \stackrel{b_{f(i)}}{\rightarrow} \beta_{2 f(i)} \beta_{1} \beta_{0}$ such that $\alpha_{2 i} \alpha_{1} \gamma_{0} \underset{R_{\mathbf{T}}}{\longleftrightarrow} * \beta_{2 f(i)} \beta_{1}$. We now have the match,

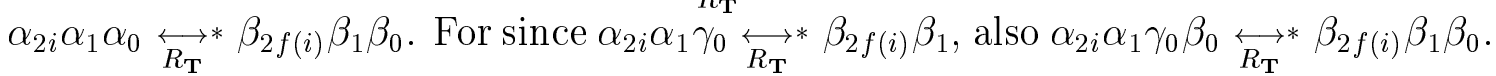
Since $\left(\alpha_{0}, \gamma_{0} \beta_{0}\right) \in R_{\mathbf{T}}$ we then have $\alpha_{2 i} \alpha_{1} \alpha_{0} \overleftrightarrow{R_{\mathbf{T}}} * \beta_{2 f(i)} \beta_{1} \beta_{0}$, as was to be shown. Finding a match for any $Y_{1} \beta_{1} \beta_{0} \stackrel{b_{j}}{\rightarrow} \beta_{2 j} \beta_{1} \beta_{0}$ is entirely similar.

Corollary 3 For any normed system of BPA equations in GNF $\triangle$ and $\alpha, \beta \in \operatorname{Var}^{*}$ it is decidable whether $\alpha \operatorname{sim} \beta$. 
Proof: A decision procedure goes as follows: Enumerate all the finitely many tableaux for $\alpha=\beta$ (this is possible by Corollary 2). By Theorem 3 $\alpha \operatorname{sim} \beta$ iff we find a successful tableau.

\section{An equational theory}

Besides yielding a straightforward decision procedure, the tableau technique can also be used to build a (weakly) sound and complete sequent-based equational theory for bisimulation equivalence of normed BPA processes given in 3-GNF. For all that is required is a family of sound rules that permit one to derive the roots of successful tableaux. The equational theory is somewhat non-standard in the arena of process algebras. As it depends on assumptions, it is different in style both from Milner's elegant equational theory for regular processes with an explicit fixed point operator $\mu$ [26] and the version in [4] without $\mu$.

Since the theory is based on the tableau system from the previous section, we restrict our attention to normed systems of process equations in 3-GNF. Let $\Delta$ be such a system. The proof system appeals to assumptions of the form $X \alpha=Y \beta$. The basic sequent of the system has the form $\Gamma \vdash_{\Delta} E=F$ where $\Gamma$ is a set of assumptions and $E, F$ range over BPA expressions. A sequent is interpreted as follows:

Definition 10 We write $\Gamma \models_{\Delta} E=F$ when it is the case that if the relation $\{(X \alpha, Y \beta) \mid X \alpha=Y \beta \in \Gamma\} \cup\left\{\left(X_{i}, E_{i}\right) \mid X_{i} \stackrel{\text { def }}{=} E_{i} \in \Delta\right\}$ is part of a bisimulation then $E$ simF.

Thus, the special case $\emptyset \models_{\Delta} E=F$ states that $E \operatorname{sim} F$ (relative to the system of process equations $\Delta$ ).

The proof system is given in Table 2. Equivalence and congruence rules are R1-5. The rules R6-10 correspond to the BPA laws of [4]. R11 and R12 deal with recursion and have been dictated by the tableau method. R11 is an assumption introduction rule, justified by the interpretation of sequents described above. R12 is an assumption elimination or discharge rule, which at the same time is a version of fixed point induction. Notice that the rule is contextual in character, involving the BPA contexts [ ] $\alpha$ and [ ] $\beta$ where [] is a 'hole'.

Definition $11 A$ proof of $\Gamma \vdash_{\Delta} E=F$ is a finite proof tree with the root labelled by $\Gamma \vdash_{\Delta} E=F$, with leaves that are instances of the axioms R1,R610 or R11 and such that the parent of a set of nodes is determined by an 


\section{Equivalence}

$\mathrm{R} 1$

$$
\Gamma \vdash_{\Delta} E=E
$$

R2

$$
\frac{\Gamma \vdash_{\Delta} E=F}{\Gamma \vdash_{\Delta} F=E}
$$

R3

$$
\frac{\Gamma \vdash_{\Delta} E=F \quad \Gamma \vdash_{\Delta} F=G}{\Gamma \vdash_{\Delta} E=G}
$$

\section{Congruence}

R4

$$
\frac{\Gamma \vdash_{\Delta} E_{1}=F_{1} \quad \Gamma \vdash_{\Delta} E_{2}=F_{2}}{\Gamma \vdash_{\Delta} E_{1}+E_{2}=F_{1}+F_{2}}
$$

R5

$$
\frac{\Gamma \vdash_{\Delta} E_{1}=F_{1} \quad \Gamma \vdash_{\Delta} E_{2}=F_{2}}{\Gamma \vdash_{\Delta} E_{1} E_{2}=F_{1} F_{2}}
$$

BPA axioms

$$
\begin{array}{lr}
\text { R6 } & \Gamma \vdash_{\Delta} E+F=F+E \\
\text { R7 } & \Gamma \vdash_{\Delta}(E+F)+G=E+(F+G) \\
\text { R8 } & \Gamma \vdash_{\Delta} E+E=E \\
\text { R9 } & \Gamma \vdash_{\Delta}(E+F) G=E G+F G \\
\text { R10 } & \Gamma \vdash_{\Delta} E(F G)=(E F) G
\end{array}
$$

\section{Recursion}

$\mathrm{R} 11$

$$
\Gamma, X \alpha=Y \beta \vdash_{\Delta} X \alpha=Y \beta
$$

R12

$$
\frac{\Gamma, X \alpha=Y \beta \vdash_{\Delta} E \alpha=F \beta}{\Gamma \vdash_{\Delta} X \alpha=Y \beta} \quad X \stackrel{\text { def }}{=} E, Y \stackrel{\text { def }}{=} F \in \Delta
$$

Table 2: Rules of inference in the equational theory 
application of one of the rules $\mathrm{R} 2-5$ or $\mathrm{R} 12$. If $\Gamma \vdash_{\Delta} E=F$ has a proof we simply write $\Gamma \vdash_{\Delta} E=F$.

In our proof that the equational theory is weakly sound and complete it turns out to be easiest to prove that it is in fact strongly sound. We need to appeal to the standard characterization of the maximal strong bisimulation as a limit:

Definition 12 For any transition graph $T=(\operatorname{Pr}$, Act,$\rightarrow)$ define the family of binary relations $\left\{\operatorname{sim}_{n}\right\}_{n=0}^{\omega}$ over $\operatorname{Pr}$ inductively as follows.

- $p \operatorname{sim}_{0} q$ for all $p, q \in P r$,

- $p \operatorname{sim}_{n+1} q$ iff

- if $p \stackrel{a}{\rightarrow} p^{\prime}$ then $\exists q^{\prime}$ with $q \stackrel{a}{\rightarrow} q^{\prime}$ and $p^{\prime} \operatorname{sim}_{n} q^{\prime}$ and

- if $q \stackrel{a}{\rightarrow} q^{\prime}$ then $\exists p^{\prime}$ with $p \stackrel{a}{\rightarrow} p^{\prime}$ and $p^{\prime} \operatorname{sim}_{n} q^{\prime}$.

Theorem 4 [27] For any image-finite transition graph we have

$$
\operatorname{sim}=\bigcap_{n=0}^{\omega} \operatorname{sim}_{n}
$$

Theorem 5 If $\Gamma \vdash_{\Delta} X \alpha=Y \beta$ then $\Gamma \models_{\Delta} X \alpha=Y \beta$

ProOf: We proceed by contraposition. Assume that we have a proof of $\Gamma \vdash_{\Delta} X \alpha=Y \beta$ but that $\Gamma \models_{\Delta} X \alpha=Y \beta$. Then $\{(X \alpha, Y \beta) \mid X \alpha=Y \beta \in$ $\Gamma\} \cup\left\{\left(X_{i}, E_{i}\right) \mid X_{i} \stackrel{\text { def }}{=} E_{i} \in \Delta\right\}$ is part of a bisimulation but $X \alpha / \operatorname{sim} Y \beta$; consequently, as $\Delta$ defines an image-finite transition graph, by Theorem 4 we know that $X \alpha / \operatorname{sim}_{n} Y \beta$ for some $n$.

Observe that if we ignore the hypotheses, then the rules preserve $\operatorname{sim}_{n}$ and all formulae that are instances of axioms other than R11 are true for all $\operatorname{sim}_{n}$. For instance, for R12 we have that if $E \alpha \operatorname{sim}_{n} F \beta$ then $X \alpha \operatorname{sim}_{n} Y \beta$ because $X \stackrel{\text { def }}{=} E \in \Delta$ and $Y \stackrel{\text { def }}{=} F \in \Delta$. Similarly, for R3 we have that $E \operatorname{sim}_{n} F$ and $F \operatorname{sim}_{n} G$ imply that $E \operatorname{sim}_{n} G$. Now significantly, in the case of R5 we can strengthen this to say that $E_{1} \operatorname{sim}_{n} F_{1}$ and $E_{2} \operatorname{sim}_{n-1} F_{2}$ imply that $E_{1} E_{2} \operatorname{sim}_{n} F_{1} F_{2}$ (when $\left|E_{1}\right|>0$.)

Now consider the proof tree for $\Gamma \vdash_{\Delta} X \alpha=Y \beta$. Since $X \alpha / \operatorname{sim}_{n} Y \beta$, by the above observations, there is a path $\pi$ to some leaf in the proof tree such that for every node $\Gamma_{i} \vdash_{\Delta} \alpha_{i}=\beta_{i}$ along $\pi$ we have $\alpha_{i} / \operatorname{sim}_{k_{i}} \beta_{i}$ for some $k_{i}$. For each $i$ choose $k_{i}$ such that it is the least number with this property. 
What could the leaf of the path look like? It cannot be an assumption in $\Gamma$, since $\Gamma$ is part of a bisimulation. Nor can it be an identity. The only other possibility is that the leaf at the end of the path $\pi$ is an instance of R11 of the form $\Gamma_{m}, X_{m}^{\prime} \alpha_{m}^{\prime}=Y_{m}^{\prime} \beta_{m}^{\prime} \vdash_{\Delta} X_{m}^{\prime} \alpha_{m}^{\prime}=Y_{m}^{\prime} \beta_{m}^{\prime}$ and such that $X_{m}^{\prime} \alpha_{m}^{\prime} /$ $\operatorname{sim}_{k_{m}} Y_{m}^{\prime} \beta_{m}^{\prime}$ where $k_{m}$ is the least number with this property. Assume that $X_{m}^{\prime} \stackrel{\text { def }}{=} E^{\prime} \in \Delta$ and $Y_{m}^{\prime} \stackrel{\text { def }}{=} F^{\prime} \in \Delta$.

As $X_{m}^{\prime} \alpha_{m}^{\prime}=Y_{m}^{\prime} \beta_{m}^{\prime}$ has been eliminated as a hypothesis in the course of the proof, there must be an application of R12 on $\pi$ with premise $\Gamma_{i} \vdash_{\Delta} E^{\prime} \alpha_{m}^{\prime}=$ $F^{\prime} \beta_{m}^{\prime}$ somewhere on the path $\pi$. On the subpath between this premise and the leaf $\Gamma_{m}, X_{m}^{\prime} \alpha_{m}^{\prime}=Y_{m}^{\prime} \beta_{m}^{\prime} \vdash_{\Delta} X_{m}^{\prime} \alpha_{m}^{\prime}=Y_{m}^{\prime} \beta_{m}^{\prime}$ there must be at least one application of the congruence rule R5 in order to build up the expressions $E^{\prime}$ and $F^{\prime}$. By the above observation on the soundness of R5 w.r.t. sim $_{n}$ every node $\alpha_{i}=\beta_{i}$ on the subpath must have $\alpha_{i} \operatorname{sim}_{k} \beta_{i}$ for all $k<k_{m}$. Since $E^{\prime}$ and $F^{\prime}$ are guarded, in at least one application of R5 an equation derived from $X_{m}^{\prime} \alpha_{m}^{\prime}=Y_{m}^{\prime} \beta_{m}^{\prime}$ must be the right-hand premise (possibly simply to introduce action prefixes). Thus we must in fact have that $E^{\prime} \alpha_{m}^{\prime} \operatorname{sim}_{k} F^{\prime} \beta_{m}^{\prime}$ for some $k \geq k_{m}$. But this then implies that $X_{m}^{\prime} \alpha_{m}^{\prime} \operatorname{sim}_{k} Y_{m}^{\prime} \beta_{m}^{\prime}$ for some $k \geq k_{m}$, contradicting our assumption that $X_{m}^{\prime} \alpha_{m}^{\prime} / \operatorname{sim}_{k_{m}} Y_{m}^{\prime} \beta_{m}^{\prime}$.

The completeness proof depends on simulating the tableau construction using the proof rules. The thinning rule usually found in sequent-based proof systems is a derived rule in ours.

Lemma 2 (Thinning) If $\Gamma \vdash_{\Delta} E=F$ then $\Gamma, \Gamma^{\prime} \vdash_{\Delta} E=F$ for any $\Gamma^{\prime}$.

The completeness proof rests on a number of lemmas and definitions which tell us how to determine our sets of hypotheses throughout a proof of $X \alpha \operatorname{sim} Y \beta$ from a successful tableau for $X \alpha \operatorname{sim} Y \beta$.

Definition 13 In a successful tableau $\mathbf{T}$, we define the set of companion nodes $\operatorname{Com}\left(E \alpha^{\prime}=F \beta^{\prime}\right)$ for a node $E \alpha^{\prime}=F \beta^{\prime}$ as the set of nodes along the path to the root of $\mathbf{T}$ that correspond to an instance of a successful terminal for termination condition 1.

For any subtableau $\mathbf{T}^{\prime}$ of $\mathbf{T}$ the set Basic $\mathbf{T}_{\mathbf{T}^{\prime}}\left(E \alpha^{\prime}=F \beta^{\prime}\right)$ for a node $E \alpha^{\prime}=$ $F \beta^{\prime}$ in $\mathbf{T}^{\prime}$ is the set of basic nodes on the path starting above $E \alpha^{\prime}=F \beta^{\prime}$ and ending at the root of $\mathbf{T}^{\prime}$.

Proposition 7 For any node $E \alpha^{\prime}=F \beta^{\prime}$ in a successful tableau $\mathbf{T}$ we have

$$
\operatorname{Com}\left(E \alpha^{\prime}=F \beta^{\prime}\right) \subseteq \operatorname{Basic}_{\mathbf{T}}\left(E \alpha^{\prime}=F \beta^{\prime}\right)
$$


Lemma 3 Let $\mathbf{T}^{\prime}$ be a subtableau of a successful tableau $\mathbf{T}$ such that $\mathbf{T}^{\prime}$ is built using only basic steps, has root $X^{\prime} \alpha^{\prime}=Y^{\prime} \beta^{\prime}$ and leaves $\alpha_{1}=\beta_{1}, \ldots, \alpha_{n}=$ $\beta_{n}$. If for some $\Gamma$ we have $\Gamma \vdash_{\Delta} \alpha_{i}=\beta_{i}$ for $1 \leq i \leq n$ then $\Gamma \vdash_{\Delta} X^{\prime} \alpha^{\prime}=Y^{\prime} \beta^{\prime}$ with a proof tree with nodes of the form $\Gamma$, Basic $\mathbf{T}^{\prime}\left(E^{\prime \prime} \alpha^{\prime \prime}=F^{\prime \prime} \beta^{\prime \prime}\right) \vdash_{\Delta} E^{\prime \prime} \alpha^{\prime \prime}=$ $F^{\prime \prime} \beta^{\prime \prime}$ for any node $E^{\prime \prime} \alpha^{\prime \prime}=F^{\prime \prime} \beta^{\prime \prime}$ in $\mathbf{T}^{\prime}$.

Proof: Induction in $d$, the depth w.r.t. basic steps of $\mathbf{T}^{\prime}$.

$d=1: \mathbf{T}^{\prime}$ consists of one basic step:

$$
\begin{aligned}
& \frac{X^{\prime} \alpha^{\prime}=Y^{\prime} \beta^{\prime}}{\left(\sum_{i=1}^{m} a_{i} \alpha_{i}\right) \alpha^{\prime}=\left(\sum_{j=1}^{n} b_{j} \beta_{j}\right) \beta^{\prime}} \quad \text { REC }
\end{aligned}
$$

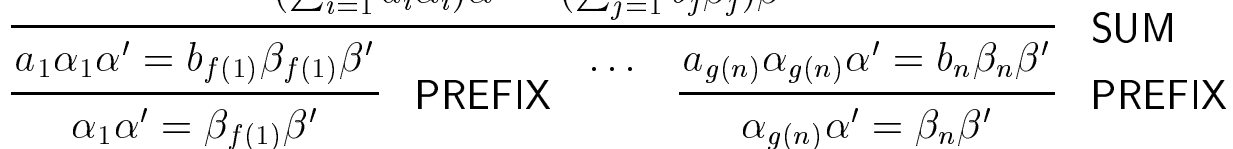

where $\Gamma \vdash_{\Delta} \alpha_{i} \alpha^{\prime}=\beta_{f(i)} \beta^{\prime}$ for $1 \leq i \leq m$ and $\Gamma \vdash_{\Delta} \alpha_{g(j)} \alpha^{\prime}=\beta_{j} \beta^{\prime}$ for $1 \leq j \leq n$. Since we have that

$$
\text { Basic }_{\mathbf{T}^{\prime}}\left(\alpha_{i} \alpha^{\prime}=\beta_{i} \beta^{\prime}\right)=\text { Basic }_{\mathbf{T}^{\prime}}\left(\alpha_{j} \alpha^{\prime}=\beta_{j} \beta^{\prime}\right)=\left\{X^{\prime} \alpha^{\prime}=Y^{\prime} \beta^{\prime}\right\}
$$

for any consequents, Lemma 2 tells us that

$$
\Gamma, X^{\prime} \alpha^{\prime}=Y^{\prime} \beta^{\prime} \vdash_{\Delta} \alpha_{i} \alpha^{\prime}=\beta_{f(i)} \beta^{\prime} \text { for } 1 \leq i \leq m
$$

and

$$
\Gamma, X^{\prime} \alpha^{\prime}=Y^{\prime} \beta^{\prime} \vdash_{\Delta} \alpha_{g(j)} \alpha^{\prime}=\beta_{j} \beta^{\prime} \text { for } 1 \leq j \leq n
$$

Repeated use of R5 followed by repeated use of R4 gives us

$$
\Gamma, X^{\prime} \alpha^{\prime}=Y^{\prime} \beta^{\prime} \vdash_{\Delta}\left(\sum_{i=1}^{m} a_{i} \alpha_{i}\right) \alpha^{\prime}=\left(\sum_{j=1}^{m} b_{j} \beta_{j}\right) \beta^{\prime}
$$

Finally, by R12 we get $\Gamma \vdash_{\Delta} X^{\prime} \alpha^{\prime}=Y^{\prime} \beta$.

Step (assuming for $d$ ): The first basic step of the subtableau is as in the base case. By induction hypothesis we have that

$$
\Gamma \vdash_{\Delta} \alpha_{i} \alpha^{\prime}=\beta_{f(i)} \beta^{\prime} \text { for } 1 \leq i \leq m
$$


and

$$
\Gamma \vdash_{\Delta} \alpha_{g(j)} \alpha^{\prime}=\beta_{j} \beta^{\prime} \text { for } 1 \leq j \leq n
$$

And by Lemma 2 we get that

$$
\Gamma, X^{\prime} \alpha^{\prime}=Y^{\prime} \beta^{\prime} \vdash_{\Delta} \alpha_{i} \alpha^{\prime}=\beta_{f(i)} \beta^{\prime} \text { for } 1 \leq i \leq m
$$

and

$$
\Gamma, X^{\prime} \alpha^{\prime}=Y^{\prime} \beta^{\prime} \vdash_{\Delta} \alpha_{g(j)} \alpha^{\prime}=\beta_{j} \beta^{\prime} \text { for } 1 \leq j \leq n
$$

The proof now proceeds as for the base case.

Lemma 4 Given a successful tableau $\mathbf{T}$, for any $X \alpha=Y \beta$ that is a terminal or the root of an eliminating subtableau we have $\operatorname{Com}(X \alpha=Y \beta) \vdash_{\Delta} X \alpha=$ $Y \beta$.

Proof: Induction in the structure of $\mathbf{T}$.

Base case $-X \alpha=Y \beta$ is a terminal: $X \alpha=Y \beta$ is either a terminal due to termination condition 2 or termination condition 1 . In the former case, R1 immediately gives us $\operatorname{Com}(X \alpha=Y \beta) \vdash_{\Delta} X \alpha=Y \beta$. In the latter case, $X \alpha=Y \beta \in \operatorname{Com}(X \alpha=Y \beta)$ so we get desired result by R11.

Step: Now $X \alpha=Y \beta$ is root of the subtableau $\mathbf{T}^{\prime}$ :

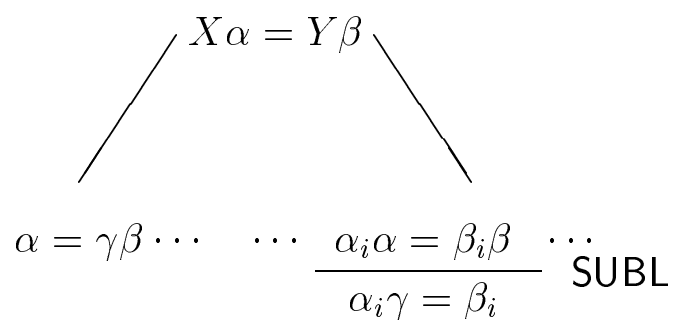

By induction hypothesis, we have $\operatorname{Com}(\alpha=\gamma \beta) \vdash_{\Delta} \alpha=\gamma \beta$ and for any SUB consequent (assume wlog that it is SUBL) $\operatorname{Com}\left(\alpha_{i} \gamma=\beta_{i}\right) \vdash_{\Delta} \alpha_{i} \gamma=\beta_{i}$. But since there are no terminals within $\mathbf{T}^{\prime}$ we have that $\operatorname{Com}(\alpha=\gamma \beta)=$ $\operatorname{Com}\left(\alpha_{i} \gamma=\beta_{i}\right)$. By R1, we get $\operatorname{Com}(\alpha=\gamma \beta) \vdash_{\Delta} \beta=\beta$ and by R5 $\operatorname{Com}(\alpha=\gamma \beta) \vdash_{\Delta} \quad \alpha_{i} \gamma \beta=\beta_{i} \beta$. By R3 this implies $\operatorname{Com}(\alpha=\gamma \beta) \vdash_{\Delta}$ $\alpha_{i} \alpha=\beta_{i} \beta$. By Lemma 3 we then get $\operatorname{Com}(X \alpha=Y \beta) \vdash_{\Delta} X \alpha=Y \beta$ as desired. Note also that by Lemma 3 that for any node $E^{\prime \prime} \alpha^{\prime \prime}=F^{\prime \prime} \beta^{\prime \prime}$ in $\mathbf{T}^{\prime}$ we have $\operatorname{Basic}_{\mathbf{T}^{\prime}}\left(E^{\prime \prime} \alpha^{\prime \prime}=F^{\prime \prime} \beta^{\prime \prime}\right) \vdash_{\Delta} E^{\prime \prime} \alpha^{\prime \prime}=F^{\prime \prime} \beta^{\prime \prime}$. 
Theorem 6 If $X \alpha \operatorname{sim} Y \beta$ (with respect to $\Delta$ ) then $\emptyset \vdash_{\Delta} X \alpha=Y \beta$

Proof: By Theorem 3 we know that $X \alpha=Y \beta$ has a successful tableau T. For each node $E^{\prime \prime} \alpha^{\prime \prime}=F^{\prime \prime} \beta^{\prime \prime}$ in $\mathbf{T}$ we have that $\operatorname{Basic}_{\mathbf{T}}\left(E^{\prime \prime} \alpha^{\prime \prime}=F^{\prime \prime} \beta^{\prime \prime}\right) \vdash$ $E^{\prime \prime} \alpha^{\prime \prime}=F^{\prime \prime} \beta^{\prime \prime}$. If $E^{\prime \prime} \alpha^{\prime \prime}=F^{\prime \prime} \beta^{\prime \prime}$ is a subtableau root or a terminal, this follows from Lemma 4, Proposition 7 and Lemma 2. If $E^{\prime \prime} \alpha^{\prime \prime}=F^{\prime \prime} \beta^{\prime \prime}$ is a node in an eliminating subtableau, it follows from the remarks at the end of the proof of Lemma 4 and Lemma 2. Since $\operatorname{Basic}_{\mathbf{T}}(X \alpha=Y \beta)=\emptyset$, the result follows.

\section{$5 \quad$ Extracting fundamental relations}

In Section 3 we have seen that the tableau system presented generates a self-bisimulation in case of successful termination. In this section we show another relationship with the work of Caucal [8] in that we give an auxiliary tableau system for extracting a fundamental relation $R$ from a successful tableau for $X \alpha=Y \beta$ with the property that $X \alpha \overleftrightarrow{{ }_{R}} * Y \beta$.

Definition 14 [8] A relation $R \subseteq \operatorname{Var}^{+} \times \operatorname{Var}^{+}$is called fundamental iff

1. $\operatorname{Dom}(R) \subseteq \operatorname{Var}, \operatorname{Im}(R) \subseteq(\operatorname{Var} \backslash \operatorname{Dom}(R))^{+}$

2. $R$ is a function: $\alpha R \beta$ and $\alpha R \gamma$ implies $\beta=\gamma$

3. $\alpha R \beta$ implies $|\alpha|=|\beta|$

From the first condition above it is immediately seen that fundamental relations are finite and from the third condition one sees that there are finitely many fundamental relations for any normed BPA process (since there are only finitely many elements of $\operatorname{Var}^{*}$ with any given norm). Seen as a rewrite relation, if $R$ is fundamental then it is also canonical, i.e. confluent and well-founded (this follows from the functionality of $R$ and the finiteness of $\operatorname{Dom}(R)$ ), and thus its least congruence is decidable.

One can think of the least congruence $\overleftrightarrow{R}^{*}$ of a relation $R$ as the set of equations provable within equational logic (with added congruence rules) using $R$ as axioms. Thus, we can we view a fundamental relation with the above property as constituting a 'local axiomatization' of sim, relative to $\Delta$ and the root equation $X \alpha=Y \beta$.

Throughout the following we shall assume the existence of a successful tableau $\mathbf{T}$ for $X \alpha=Y \beta$. 
The fundamental observation is that for the eliminating subtableau for $X \alpha=Y \beta$ we must, when $\alpha=\gamma \beta$ is the residual, have $Y \beta \operatorname{sim} X \gamma \beta$ and thus by Proposition $1 Y \operatorname{sim} X \gamma$; assume now wlog that $X$ and $Y$ are not the same variable. Since $|Y|=|X \gamma|$ we know that $Y$ does not occur in $X \gamma$, so $(Y, X \gamma)$ is a fundamental relation. Clearly, if we let $R=\{(\alpha, \gamma \beta),(Y, X \gamma)\}$ we have $X \alpha \underset{R}{\longleftrightarrow} * Y \beta$. The auxiliary tableau system now gradually modifies and extends $R$ until it becomes a fundamental relation with this property. While doing this we may need to introduce new goals.

The auxiliary tableau system is built around sequents of the form $R \vdash_{\mathbf{T}} \Gamma$ where $R$ is a finite subset of $\operatorname{Var} \times \operatorname{Var}^{+}$and $\Gamma$ is a finite set of equations over Var*. Since the relations $R$ constructed are all fundamental (by Proposition 9 below), they are all confluent and strongly normalizing, so for any $\alpha$ its unique normal form $\alpha \downarrow R$ is known to exist.

At all times during the auxiliary tableau construction we rewrite as much of $\Gamma$ as much as possible using $R$. We may then need to introduce new goals or extend $R$. There are in general three possible situations possibly at any point where this can happen:

- If an equation $X \alpha=Y \beta$ has the residual $\alpha=\gamma \beta$ and $R \cup\{(Y, X \gamma)\}$ is fundamental we simply extend $R$ with the pair $(Y, X \gamma)$. This justifies the rule EXTEND.

- If an equation $X \alpha=Y \beta$ has the residual $\alpha=\gamma \beta$ but $R \cup\{(Y, X \gamma)\}$ is not fundamental because $Y \in \operatorname{Dom}(R)$ with $\left(Y, X_{1} \gamma_{1}\right) \in R$ for some $X_{1} \gamma_{1}$. Then we must also compare $X_{1} \gamma_{1}$ and $X \gamma$. This gives rise to the rule COMPARE.

- If an equation $X \alpha=Y \beta$ has the residual $\alpha=\gamma \beta$ but $R \cup\{(Y, X \gamma)\}$ is not fundamental even though $Y \notin \operatorname{Dom}(R)$ because some variable $Z \in \operatorname{Dom}(R)$ occurs in $X \gamma$. We must rewrite $X \gamma$ and can then add $(Y,(X \gamma) \downarrow R)$ to $R$. This is the basis of the rule UPDATE.

The rule REWRITE tells us that we must rewrite using $R$ whenever possible. And finally there are the rules CONGL and CONGR whose purpose is to remove identical heads and tails from equations. CONGR is strictly speaking not necessary but has been included for reasons of symmetry.

The rules have the following priority: CONGL must always be used whenever possible to remove identical leftmost variables. Next in priority is REWRITE. Finally, the other rules have equal priority. Thus we see that REWRITE will only be used between updatings of $R$, as desired.

The rules in Table 3 are sound w.r.t. sim. 


$$
\begin{aligned}
& \begin{array}{cl}
\text { EXTEND } & R \vdash_{\mathbf{T}} X \alpha=Y \beta, \Gamma \\
R, Y=X \gamma \vdash_{\mathbf{T}} \alpha=\gamma \beta, \Gamma & \text { if } \alpha=\gamma \beta \text { is the residual of } \\
X \alpha=Y \beta \text { and } R, Y=X \gamma \text { is }
\end{array} \\
& \text { fundamental } \\
& \text { COMPARE } \frac{R \vdash_{\mathbf{T}} X \alpha=Y \beta, \Gamma}{R \vdash_{\mathbf{T}} X_{1} \gamma_{1}=X \gamma, \alpha=\gamma \beta, \Gamma} \\
& \text { if } Y \in \operatorname{Dom}(R) \text { and } \alpha=\gamma \beta \text { is } \\
& \left(Y, X_{1} \gamma_{1}\right) \in R \\
& \text { UPDATE } \frac{R \vdash_{\mathbf{T}} X \alpha=Y \beta, \Gamma}{R, Y=(X \gamma) \downarrow R \vdash_{\mathbf{T}}(\alpha) \downarrow R=(\beta) \downarrow R, \Gamma} \\
& \text { if } Y \notin \operatorname{Dom}(R) \text { and } \alpha=\gamma \beta \text { is } \\
& \text { some variable } Z \in \operatorname{Dom}(R) \\
& \text { occurs in } X \gamma \\
& \begin{array}{lll}
\text { REWRITE } & \frac{R \vdash_{\mathbf{T}} X \alpha=Y \beta, \Gamma}{} & \text { if }(X \alpha) \downarrow R \neq X \alpha \text { or } \\
& R \vdash_{\mathbf{T}}(X \alpha) \downarrow R=(Y \beta) \downarrow R, \Gamma & (Y \beta) \downarrow R \neq Y \beta
\end{array} \\
& \begin{array}{ll}
\text { CONGL } & \frac{R \vdash_{\mathbf{T}} \alpha \delta_{1}=\alpha \delta_{2}, \Gamma}{R \vdash_{\mathbf{T}} \delta_{1}=\delta_{2}, \Gamma} \\
\text { CONGR } & \frac{R \vdash_{\mathbf{T}} \alpha_{1} \delta=\alpha_{2} \delta, \Gamma}{R \vdash_{\mathbf{T}} \alpha_{1}=\alpha_{2}, \Gamma}
\end{array}
\end{aligned}
$$$$
\text { the residual for } X \alpha=Y \beta \text { but }
$$$$
\text { the residual for } X \alpha=Y \beta \text { but }
$$

Table 3: Rules of the auxiliary tableau system 
Definition 15 A relation $R \subseteq \operatorname{Var} \times \operatorname{Var}^{+}$is said to be sim-consistent if $R \subseteq$ sim. Similarly, a set of equations $\Gamma$ is called sim-consistent if for all $\alpha^{\prime}=\beta^{\prime} \in \Gamma^{\prime}$ we have $\alpha^{\prime} \operatorname{sim} \beta^{\prime}$.

Proposition 8 (Soundness under $\operatorname{sim}$ ) If $R^{\prime} \vdash_{\mathrm{T}} \Gamma^{\prime}$ has $R^{\prime}$ and $\Gamma^{\prime}$ simconsistent then for the consequent $R^{\prime \prime} \vdash_{\mathrm{T}} \Gamma^{\prime \prime}$ of any rule application to $R^{\prime} \vdash_{\mathrm{T}}$ $\Gamma^{\prime}, R^{\prime \prime}$ and $\Gamma^{\prime \prime}$ are sim-consistent.

The rules also preserve fundamentality:

Proposition 9 If in $R^{\prime} \vdash_{\mathrm{T}} \Gamma^{\prime}$ we have that $R^{\prime}$ is fundamental and simconsistent and $\Gamma$ is sim-consistent, then for the consequent $R^{\prime \prime} \vdash_{\mathrm{T}} \Gamma^{\prime \prime}$ of any rule application to $R^{\prime} \vdash_{\mathrm{T}} \Gamma^{\prime}$ we have that $R^{\prime \prime}$ is fundamental.

We can now be precise about the notion of an auxiliary tableau.

Definition 16 An auxiliary tableau for $R \vdash_{\mathrm{T}} \Gamma$ is a maximal sequence of sequents $R_{0} \vdash_{\mathbf{T}} \Gamma_{0}, R_{1} \vdash_{\mathbf{T}} \Gamma_{1}, \ldots, R_{n-1} \vdash_{\mathbf{T}} \Gamma_{n-1}, R_{n} \vdash_{T} \Gamma_{n}$ where $R \vdash_{\mathbf{T}} \Gamma=$ $R_{0} \vdash_{\mathrm{T}} \Gamma_{0}$ and for all $i \geq 0 R_{i+1} \vdash_{\mathrm{T}} \Gamma_{i+1}$ is the consequent of using a rule in Table 3 with $R_{i} \vdash_{\mathrm{T}} \Gamma_{i}$ as premise. An auxiliary tableau is finite if for some $n$ all equations in $\Gamma_{n}$ are identities (i.e. of the form $\alpha^{\prime}=\alpha^{\prime}$ for some $\alpha^{\prime}$ ).

Lemma 5 If $\Gamma$ is sim-consistent all auxiliary tableaux for $\emptyset \vdash_{\mathrm{T}} \Gamma$ are finite.

Proof: Every time an equation $\alpha^{\prime}=\beta^{\prime}$ is replaced in a rule application, it is replaced by equations whose norms are all $\leq\left|\alpha^{\prime}\right|=\left|\beta^{\prime}\right|$. At least one of these new equations has norm $<\left|\alpha^{\prime}\right|$. Thus we must eventually reach a situation where all equations in a sequent are identities, possibly of the form $\epsilon=\epsilon$.

Theorem 7 If $R_{0}$ is fundamental and sim-consistent and $\Gamma_{0}$ is sim-consistent,

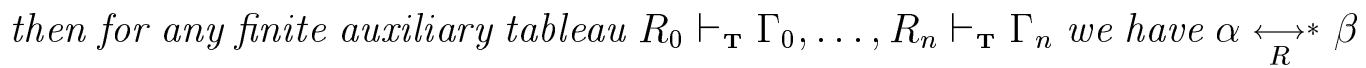
with $R=R_{n}$ for any $\alpha=\beta \in \Gamma_{0}$.

PROOF: We proceed by induction in $n$.

$n=1$ : The auxiliary tableau is $R_{0} \vdash_{\mathbf{T}} \Gamma, R \vdash_{\mathbf{T}} \Gamma^{\prime}$, and every equation $\alpha=\beta$

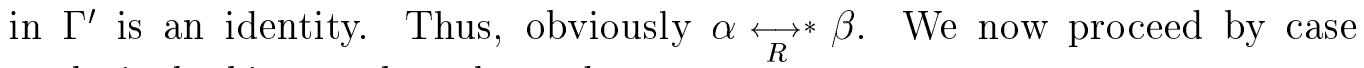
analysis, looking at the rule used.

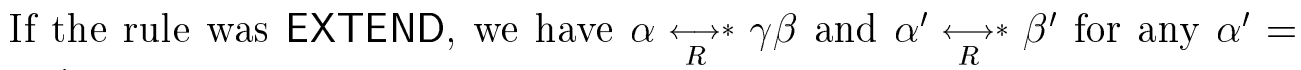
$\beta^{\prime} \in \Gamma^{\prime}$. Therefore, $X \alpha \overleftrightarrow{R} * X \gamma \beta$ and $X \alpha \overleftrightarrow{R} * Y \beta$ as desired.

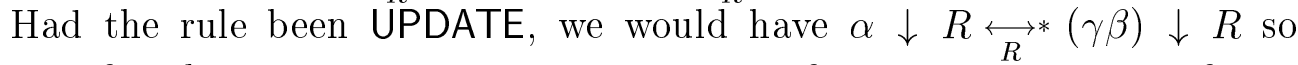

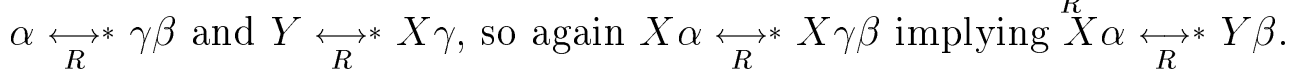


If the rule was REWRITE, we would have $(X \alpha) \downarrow R \overleftrightarrow{R^{*}}(Y \beta) \downarrow R$ implying $X \alpha \overleftrightarrow{R} \overleftrightarrow{R}^{*} Y \beta$.

And if the rule used had been COMPARE, we had $X_{1} \gamma_{1} \overleftrightarrow{R} * X \gamma$ and

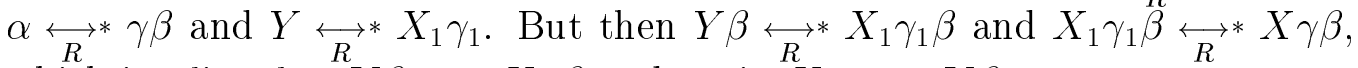
which implies that $Y \beta \overleftrightarrow{R} * X \gamma \beta$ and again $X \alpha \overleftrightarrow{R} * Y \beta$.

CONGL and CONGR are immediate.

Step: The auxiliary tableau is now $R_{0} \vdash_{\mathrm{T}} \Gamma_{0}, R_{1} \vdash_{\mathrm{T}} \Gamma_{1} \ldots, R_{n} \vdash_{\mathrm{T}} \Gamma_{n}$ and $R_{1} \vdash_{\mathbf{T}} \Gamma_{1}, \ldots, R_{n} \vdash_{\mathbf{T}} \Gamma_{n}$ is an auxiliary tableau for $R_{1} \vdash_{\mathbf{T}} \Gamma_{1}$, so for every

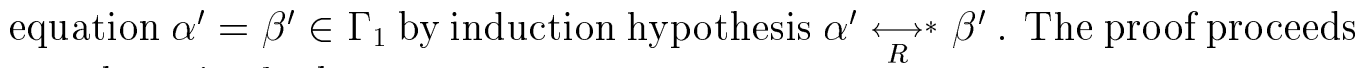
exactly as in the base case.

From the above theorem and Lemma 5 we now get the desired result:

Corollary 4 If $X \alpha$ sim $Y \beta$ by the successful tableau $\mathbf{T}$, for any auxiliary

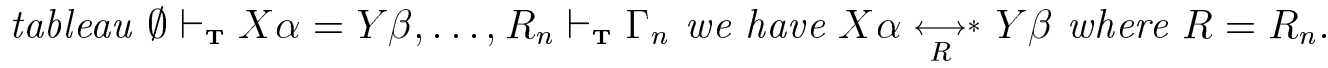

\section{Conclusions and perspectives}

In this paper we have given an alternative and much simpler proof of the decidability of bisimulation equivalence for normed BPA processes, first proved by Baeten, Bergstra and Klop [1,2] and later by Caucal [7, 8]. Our decidability proof uses a tableau system closely related to the branching algorithms employed in the study of equivalence problems in language theory [24, 15]. If a successful tableau for an equation $\alpha=\beta$ exists, the tableau provides us with a finite witness for a bisimulation containing $(\alpha, \beta)$, a self-bisimulation in the sense of $[7,8]$. The tableau method allows us to extract a sound and complete sequent-based equational theory for bisimilarity over normed BPA processes in 3-GNF. Finally, we have shown how to extract a fundamental relation $R$ (as in the work of [8]) from a successful tableau for $\alpha=\beta$ such that $\alpha \overleftrightarrow{R}$ ↔* $\beta$.

Since the first appearance of this paper, there have been a number of important developments. The present paper only considers the normed case. In [14], it is shown that bisimulation equivalence is indeed decidable for all of BPA. The decidability proof relies on showing that the maximal bisimulation of any BPA transition graph is generated by a finite self-bisimulation; the decision algorithm consists of two semi-decision procedures: one searches for such a self-bisimulation, the other tries to find a bisimulation error. The method does not yield an equational theory, nor does it give us any clues as to the complexity of the bisimulation problem. 
A first complexity analysis of the bisimulation problem was given in [23] by Huynh and Tian who showed that the complexity of the bisimilarity problem for normed BPA is in $\Sigma_{2}^{p}$ in the polynomial-time hierarchy. Later, Hirshfeld, Jerrum and Moller showed that the bisimulation problem for normed BPA is in fact in $\mathbf{P}$ [18]. Burkart, Caucal and Steffen have since shown that the bisimulation problem is elementary in the general case [6].

Recently, attention has been focused on the process calculus BPP where a non-communicating parallel (full merge) operator takes the place of sequencing. Christensen, Hirshfeld and Moller have proved [13, 12] that bisimulation equivalence by using a tableau technique similar to the one in this paper. Again, a by-product of the decision procedure is a sound and complete sequent-style equational theory for bisimilarity. The first author has shown [21] that all other known equivalences are undecidable for BPP; bisimulation equivalence thus seems to have a very special status as far as decidability is concerned. However, even when a slight extension of BPP is considered, namely that of restriction, bisimilarity also becomes undecidable [11]. An interesting question is what happens if instead BPP and BPA are combined. The resulting calculus is similar to the PA calculus considered in e.g. [4]. A partial answer to this question can be found in [10] where it is shown that bisimulation equivalence is decidable for processes in the union of normed BPP and normed BPA. Another, related result is found in [25] in which Kučera shows that bisimilarity is decidable for parallel compositions of normed BPA processes.

In Section 2.3 we noted the correspondence between BPA and contextfree grammars. There is a well-known and fundamental correspondence between context-free grammars and pushdown automata as regards language definability but this breaks down when one considers the transition graphs under the notion of bisimulation equivalence. In [9], Caucal and Monfort show that pushdown automata are more expressive than BPA in the sense that there exist pushdown automata whose transition graphs are not bisimilar to any BPA transition graph. The question of whether bisimilarity is decidable for general pushdown automata is nontrivial; it remains open at the time of writing. However, the second author has recently shown that bisimilarity is decidable for normed pushdown automata [29]. The proof of this uses a tableau technique to establish semi-decidability of bisimulation equivalence in conjunction with the well-known result that non-bisimilarity is semi-decidable.

Weak versions of behavioural equivalences arise when unobservable actions are considered. As the strong behavioural equivalences are special cases of their weak counterparts, it is clear by the results of [17] that all weak equivalences other than bisimilarity are undecidable for normed BPA 
with unobservable actions. There exist a number of weak versions of bisimilarity. In [20] it was shown by means of a tableau decision procedure that van Glabbeek's branching bisimulation equivalence [31] is decidable for normed BPA processes that cannot terminate silently. However, the question remains open for weak bisimulation equivalence even in the normed case; Proposition 1 does not hold for weak bisimilarity so a different approach must be used.

\section{References}

[1] J.C.M. Baeten, J.A. Bergstra, and J.W. Klop. Decidability of bisimulation equivalence for processes generating context-free languages. In LNCS 259, pages 93-114. Springer-Verlag, 1987.

[2] J.C.M. Baeten, J.A. Bergstra, and J.W. Klop. Decidability of bisimulation equivalence for processes generating context-free languages. Technical Report CS-R8632, CWI, September 1987.

[3] J.A. Bergstra and J.W. Klop. Process algebra for synchronous communication. Information and Control, 60:109-137, 1984.

[4] J.A. Bergstra and J.W. Klop. Process theory based on bisimulation semantics. In J.W. de Bakker, W.P de Roever, and G. Rozenberg, editors, LNCS 354, pages 50-122. Springer-Verlag, 1988.

[5] J. Bradfield and C. Stirling. Verifying temporal properties of processes. In $L N C S$ 458, pages 115-125. Springer-Verlag, 1990.

[6] O. Burkart, D. Caucal and B. Steffen An Elementary Decision Procedure for Arbitrary Context-Free Processes In Proceedings of MFCS'95, LNCS. Springer-Verlag 1995.

[7] D. Caucal. Graphes canoniques de graphes algébriques. Rapport de Recherche 872, INRIA, Juillet 1988.

[8] D. Caucal. Graphes canoniques de graphes algébriques. Informatique théorique et Applications (RAIRO), 24(4):339-352, 1990.

[9] D. Caucal and R. Monfort. On the transition graphs of automata and grammars. Proceedings of Graph-Theoretic Concepts in Computer Science, 1990

[10] I. Černá, M. Křetínský, and A. Kučera. Bisimilarity is Decidable in the Union of Normed BPA and Normed BPP Processes. In Proceedings of 
the 1st International Workshop on Verification of Infinite State Systems (INFINITY'96), pages 32-46, MIP-9614, University of Passau, 1996. Also in Electronic Notes in Theoretical Computer Science, volume 5, Elsevier, 1997.

[11] S. Christensen. Decidability and Decomposition in Process Algebras. PhD thesis, University of Edinburgh, 1993.

[12] S. Christensen, Y. Hirshfeld, and F. Moller. Bisimulation is decidable for all basic parallel processes. In Proceedings of CONCUR '93. SpringerVerlag, 1993.

[13] S. Christensen, Y. Hirshfeld, and F. Moller. Decomposability, decidability and axiomatisability for bisimulation equivalence on basic parallel processes. In Proceedings of LICS '93. IEEE, Computer Society Press, June 1993.

[14] S. Christensen, H. Hüttel, and C. Stirling. Bisimulation equivalence is decidable for all context-free processes. Information and Computation, 121:143-148, 1995.

[15] B. Courcelle. An axiomatic approach to the Korenjak-Hopcroft algorithms. Mathematical Systems Theory, 16:191-231, 1983.

[16] J.F. Groote. A short proof of the decidability of bisimulationf or normed bpa-processes. Information Processing Letters, 42:167-171, 1992.

[17] J.F. Groote and H. Hüttel. Undecidable equivalences for basic process algebra. Information and Computation, 115(2):354-371, 1994

[18] Y. Hirshfeld, M. Jerrum and F. Moller. A polynomial algorithm for deciding bisimilarity of normed context-free processes. Department of Computer Science, Report ECS-LFCS-94-286, 1994.

[19] J. Hopcroft and J.D. Ullman. Introduction to Automata Theory, Languages, and Computation. Addison-Wesley, 1979.

[20] H. Hüttel. Silence is golden: Branching bisimilarity is decidable for context-free processes. In Proceedings of CAV91. Springer-Verlag, 1991.

[21] H. Hüttel Undecidable Equivalences for Basic Parallel Processes. In Proceedings of TACS 94, Springer LNCS 789, pp. 454-464, 1994 
[22] Dung T. Huynh and Lu Tian. On Deciding Readiness and Failure Equivalences for Processes. Information and Computation 117(2): 193-205, 1995.

[23] Dung T. Huynh and Lu Tian. Deciding bisimilarity of normed contextfree processes is in $\Sigma_{2}^{p}$. Technical Report UTDCS-1-92, University of Texas at Dallas, January 1992. To appear in Theoretical Computer Science.

[24] A.J. Korenjak and J.E. Hopcroft. Simple deterministic languages. In Proceedings of Seventh Annual IEEE Symposium on Switching and Automata Theory, pages 36-46, 1966.

[25] A. Kučera. How to Parallelize Sequential Processes. In Proceedings of CONCUR 97, pages 302-316, Springer LNCS 1243. Springer-Verlag, 1997.

[26] R. Milner. A complete inference system for a class of regular behaviours. Journal of Computer and System Sciences, 28:439-466, 1984.

[27] R. Milner. Communication and Concurrency. Prentice-Hall International, 1989.

[28] D.M.R. Park. Concurrency and automata on infinite sequences. In P. Deussen, editor, Proceedings of 5th GI Conference LNCS 104, pages 167-183. Springer-Verlag, 1981.

[29] C. Stirling. Decidability of Bisimulation Equivalence for Normed Pushdown Processes In Proceedings of CONCUR 96, LNCS 1119, pages 217-232. Springer-Verlag, 1996.

[30] C. Stirling and D. Walker. Local model checking in the modal mucalculus. In LNCS 351, pages 369-383. Springer-Verlag, 1989.

[31] R.J. van Glabbeek. The linear time - branching time spectrum. In J.C.M. Baeten and J.W. Klop, editors, Proceedings of CONCUR 90, Amsterdam, LNCS 458, pages 278-297. Springer-Verlag, 1990. 


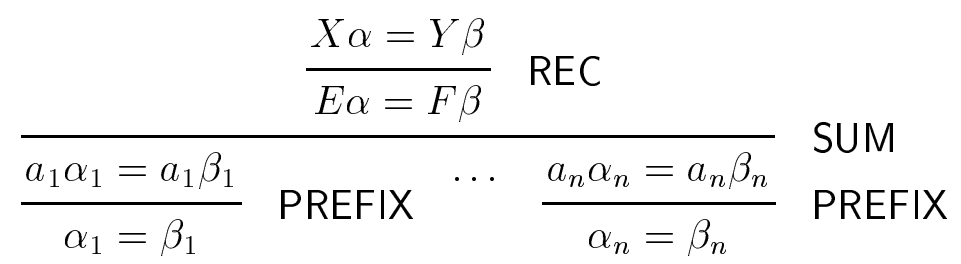

Figure 2: A basic step in the tableau system

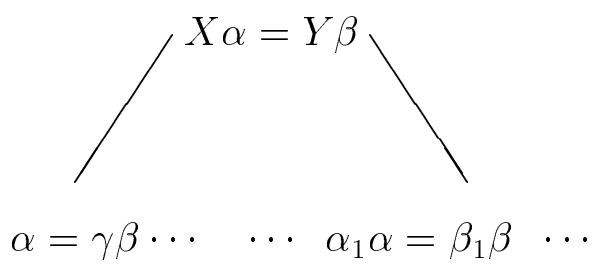

Figure 3: An eliminating subtableau for $X \alpha=Y \beta$.

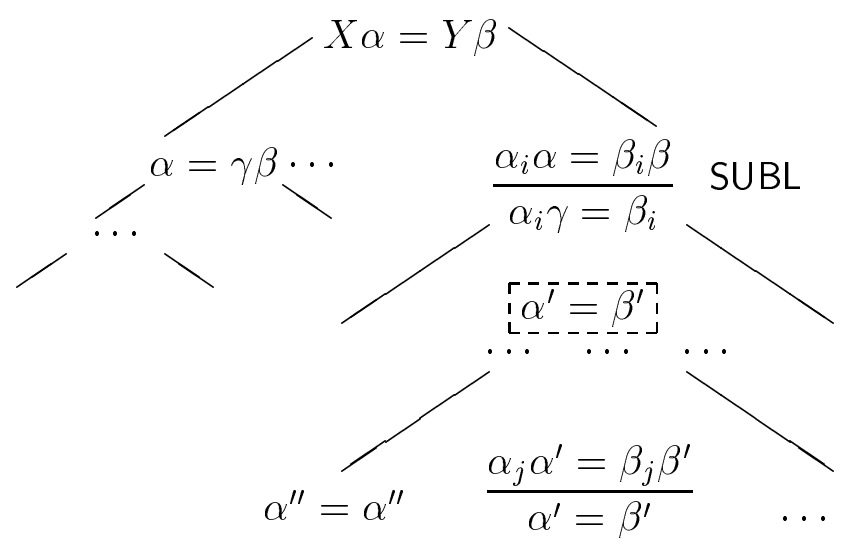

Figure 4: A tableau for $X \alpha=Y \beta$; some successful leaves are shown 


$$
\begin{aligned}
& \frac{X=A}{a Y X+b=a C+b} \text { REC }
\end{aligned}
$$

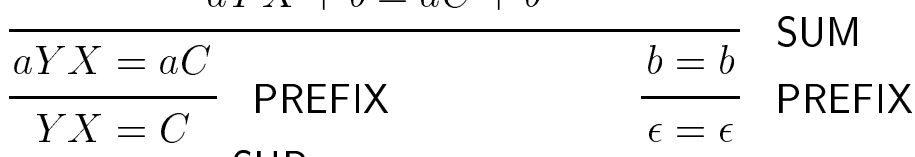

$$
\begin{aligned}
& \overline{Y X=C} \text { SUB } \\
& \begin{array}{ll}
\frac{Y X X=b A A}{X X=A A} & \text { REC } \\
\text { PREFIX }
\end{array} \\
& \begin{array}{cl}
\frac{\overline{(a Y X+b) X=(a C+b) A}}{\frac{a Y X X=a C A}{Y X X=C A}} \text { PREFIX } \frac{b X=b A}{X=A} & \text { SUM } \\
\text { PREFIX }
\end{array} \\
& Y X=C \quad \text { SUB }
\end{aligned}
$$

Figure 5: A successful tableau for $X=A$ of Example 2 